\title{
Recent Advances in Discovery of New Tyrosine Kinase Inhibitors Using Computational Methods ${ }^{+}$
}

\author{
Vesna Rastija \\ Department of Agroecology and Environmental Protection, Faculty of Agrobiotechnical Sciences Osijek, Josip \\ Juraj Strossmayer University of Osijek, 31000 Osijek, Croatia; vrastija@pfos.hr \\ + Presented at the 2nd Molecules Medicinal Chemistry Symposium (MMCS): Facing Novel Challenges in \\ Drug Discovery, Barcelona, Spain, 15-17 May 2019.
}

Published: 8 August 2019

Keywords: tyrosine kinase; molecular docking; leukemia

Tyrosine-protein kinases catalyze chemical reactions that transfer a phosphate group from adenosine triphosphate (ATP) to a tyrosine residue in a protein. Cytoplasmic/non-receptor tyrosine kinases, which act as regulatory proteins, play key roles in cell differentiation, motility, proliferation, and survival. Recent advances have implicated the role of tyrosine kinases in the pathophysiology of cancer. Selective tyrosine kinase inhibitors can block their oncogenic activation in cancer cells and can be applied as a new mode of cancer therapy. Nine Src-family tyrosine kinase have been identified, among which are c-Src and Hck.

Numerous studies have shown evidence of association between c-Src kinases and leukemia. Series of inhibitors (DSA compounds) are based on the central chemical scaffold of imatinib, a drug used for chronic myelogenous leukemia treatment. A series of new 7-chloroquinoline-arylamidine (CQArA) hybrids has been evaluated by quantitative structure-activity relationship (QSAR) analysis in order to signify the importance of structural and chemical attributes for the anticancer activity and propose new analogues with improved activity. The interaction of CQArA hybrids and c-Src in silico has been evaluated by molecular docking based on the binding mode of the DSA inhibitor. It was confirmed that the most active compound binds on the pocket between the small and large lobes of the c-SRC, mostly throughout the hydrogen bonds and van der Waals interactions. Further, an interaction of new 5-arylidenerhodanines and hematopoietic cell tyrosine kinase (Hck) in silico has been evaluated by molecular docking based on the binding mode of quercetin as an inhibitor. Excessive Hck activation is associated with several types of leukemia and enhances cell proliferation. The binding interactions of the most active compound have shown strong hydrogen bonding and van der Waals interactions with the target protein in the catalytic domain of Hck.

Finding new tyrosine kinase inhibitors and their binding modes provides the foundation for the development of new leukemia drugs.

(C) 2019 by the authors. Licensee MDPI, Basel, Switzerland. This article is an open access article distributed under the terms and conditions of the Creative Commons Attribution (CC BY) license (http://creativecommons.org/licenses/by/4.0/). 